\title{
Acute Pancreatitis Secondary to Severe Hypertriglyceridemia: Management of Severe Hypertriglyceridemia in Emergency Setting
}

\author{
Ahmad Chaudhary ${ }^{\mathrm{a}}$, Umair Iqbal ${ }^{\mathrm{a},}$, , Hafsa Anwar ${ }^{\mathrm{b}}$, Hafiz Umair Siddiquic, \\ Madiha Alvi ${ }^{\mathrm{d}}$
}

\begin{abstract}
Hypertriglyceridemia (HTG) is the third most common cause of acute pancreatitis (AP). The incidence of AP is around $10-20 \%$ with levels $>$ $2,000 \mathrm{mg} / \mathrm{dL}$. We present here a case of a 44-year-old male with history of uncontrolled diabetes mellitus and HTG admitted with severe abdominal pain. Labs revealed elevated lipase and amylase. CT of abdomen with contrast showed AP. He was found to have a triglyceride (TG) level of 6,672 mg/dL. Besides conventional treatment for AP with intravenous (IV) hydration, he was started on IV regular insulin along with dextrose saline. He had marked improvement in his TG level the next day. He was continued on insulin and dextrose saline with hourly glucose monitoring until TG was $<500 \mathrm{mg} / \mathrm{dL}$. He was discharged on statins and fenofibrate. The goal of management of AP secondary to severe HTG in emergency setting is to lower the TG levels to less than 500 as quickly as possible as lower levels are associated with good clinical outcomes. Apheresis and IV insulin are both helpful in lowering TG levels with no randomized controlled trials showing greater efficacy of one over other. Further episodes of AP can be prevented by lifestyle modification and lipid lowering drugs to keep TG levels below $500 \mathrm{mg}$ / dL. Fibrates are first-line drugs to lower TG and used either alone or in conjunction with statins. Periodic plasmapheresis can also be considered in some non-compliant patients with recurrent episodes of pancreatitis.
\end{abstract}

Keywords: Acute pancreatitis; Severe hypertriglyceridemia; Apharesis; Intravenous insulin

\section{Introduction}

Acute pancreatitis (AP) is the inflammation of the pancreas

Manuscript submitted May 10, 2017, accepted May 12, 2017

\footnotetext{
${ }^{a}$ Department of Medicine, Bassett Medical Center, Cooperstown, NY, USA bow University of Health and Sciences, Karachi, Pakistan ${ }^{\mathrm{c}}$ Cleveland Clinic Foundation, Cleveland, OH, USA

${ }^{\mathrm{d} D e p a r t m e n t}$ of Endocrinology, Bassett Medical Center, Cooperstown, NY, USA

${ }^{\mathrm{e}}$ Corresponding Author: Umair Iqbal, Department of Medicine, Bassett Medical Center, Cooperstown, NY, USA. Email: umair.iqbal@bassett.org
}

doi: https://doi.org/10.14740/gr762e characterized by abdominal pain and elevation of pancreatic enzymes. Studies show that overall mortality in patients with $\mathrm{AP}$ is around 5\% and even more in admitted patients [1]. AP has many causative risk factors, with alcohol and gallstones being the most common. Hypertriglyceridemia (HTG) is the third most common cause of AP and is responsible for almost $1-4 \%$ of AP [2-4]. Studies show that risk of AP increased many times with serum HTG levels of $>1,000 \mathrm{mg} / \mathrm{dL}$. We describe here a case of AP secondary to HTG and discuss the management of HTG in the emergency setting.

\section{Case Report}

A 44-year-old male with past medical history significant for poorly controlled diabetes mellitus, HTG, hypertension, and obesity obstructive sleep apnea non-compliant with continuous positive airway pressure presented with abdominal pain in the epigastric region radiating to the back. He also reported several episodes of vomiting, no fever or chills, no diarrhea, black or tarry stools, weight loss or night sweats. He had two episodes of pancreatitis in last couple of years and reported pain similar to these episodes. He denied excessive alcohol use and is a non-smoker. His medications include statins, fenofibrate, celecoxib, metformin, pantoprazole, insulin glargine 75 units BID and meal time insulin lispro. He had cholecystectomy in the past. His vitals were blood pressure 112/61, pulse 71 , temperature $37^{\circ} \mathrm{C}\left(98.6^{\circ} \mathrm{F}\right)$, and respiratory rate 16 . On physical exam, he was in moderate distress with abdominal pain. On abdominal exam, he had generalized tenderness, no hepatosplenomegaly, and positive bowel sound. The rest of the physical exam was unremarkable. Labs revealed lipase of $5,006 \mathrm{U} / \mathrm{L}$, amylase of $299 \mathrm{U} / \mathrm{L}$, AST of $14 \mathrm{U} / \mathrm{L}$, ALT of 28 $\mathrm{U} / \mathrm{L}$ and blood glucose of $189 \mathrm{mg} / \mathrm{dL}$. Other serum and liver chemistries were unremarkable. CT of abdomen with contrast was done and revealed moderate peri-pancreatic edema without fluid collection concerning for pancreatitis. He was started on Ringer's lactate for hydration. Triglyceride (TG) levels were checked and found to be $6,672 \mathrm{mg} / \mathrm{dL}$. Total cholesterol was $677 \mathrm{mg} / \mathrm{dL}$, low-density lipoprotein (LDL) was $36 \mathrm{mg} / \mathrm{dL}$ and high-density lipoprotein (HDL) was $20 \mathrm{mg} / \mathrm{dL}$. Given unavailability of therapeutic apheresis for correction of HTG, patient was started on insulin infusion $0.1 \mathrm{units} / \mathrm{kg} / \mathrm{h}$ along with D5W $75 \mathrm{cc} / \mathrm{h}$. His TG level came down to 3,523 the next day. 
He was continued on insulin infusion and dextrose water with hourly blood glucose check until serum TG was less than 500, which took him 8 days. He was discharged on fenofibrate and statins daily for optimal control of TG. He was also referred for genetic testing to rule out genetic disorders associated with lipid metabolism.

\section{Discussion}

HTG is defined as serum TG levels greater than $150 \mathrm{mg} / \mathrm{dL}$, with very severe cases greater than $2,000 \mathrm{mg} / \mathrm{dL}$. The incidence of AP in patients with $\mathrm{TG}>1,000$ is $5 \%$, which increases to $10-20 \%$ with levels $>2,000 \mathrm{mg} / \mathrm{dL}$ [5]. It is also recommended that all patients with levels $>500 \mathrm{mg} / \mathrm{dL}$ should undergo genetic testing to rule out genetic disorders associated with lipid metabolism. Fredrickson classified primary HTG into five classes with class I, IV and V having increased predisposition for AP [6]. Other secondary causes of HTG include diabetes, alcohol, pregnancy and variety of drugs including anti-retroviral drugs, beta-blockers and thiazides diuretics [7].

Non-pharmacological therapy includes lifestyle modification including weight loss, exercise, and restricting fat and simple sugars in the diet. Fibrates are first-line therapy in the treatment of HTG. Other drugs helpful in treatment of HTG include niacin and omega 3 fatty acids. These drugs can be used alone or in combination with statins [8]. Multiple treatment strategies have been described in the literature for severe HTG. Apheresis is usually considered the first-line strategy to treat severe HTG if available with many studies supporting its clinical utility $[9,10]$. Therapeutic plasma exchange is the modality of choice if apheresis is considered. In patients who developed an episode of AP secondary to HTG, the goal is to bring the TG levels down to $<500$ as studies show improved clinical outcomes while very high levels of TG can increase systemic inflammation associated with AP $[2,11]$. In a case series of seven patients with average TG of $1,406 \mathrm{mg} / \mathrm{dL}$, a $41 \%$ decrease in TG levels was reported with single plasma exchange [9]. In another case report, TG was lowered from 2,410 to 138 $\mathrm{mg} / \mathrm{dL}$ after 3 days of apheresis [10]. Alternatively insulin can be used to treat severe HTG in an emergency setting if apheresis is unavailable or not tolerated by the patient. Intravenous (IV) insulin is more effective than subcutaneous insulin. Standard approach is $0.1-0.3$ units $/ \mathrm{kg} / \mathrm{h}$ IV regular insulin along with dextrose saline to maintain blood glucose between 150 and $200[12,13]$. Insulin decreases TG levels by enhancing lipoprotein lipase and inhibiting hormone-sensitive lipase in adipocytes [14]. The role of heparin as adjunct to insulin is described in the literature but it is controversial and is generally not recommended $[15,16]$. The treatment of severe HTG in the emergency setting in pregnant women is also similar with plasma exchange or IV insulin. There are no randomized controlled trials which compare efficacy of insulin with apheresis in treatment of severe HTG and thus treatment is usually based upon availability and preference. The long-term goal in patients who had episodes of AP secondary to HTG is to prevent further episodes by optimizing the lipid-lowering therapy and lifestyle modifications. In patients not responsive or non- compliant to this approach, periodic apheresis can also be done to prevent future episodes of AP [17].

\section{Conclusion}

Clinicians should consider apheresis or IV insulin in settings of AP secondary to severely elevated levels of TG, as decreasing the TG early improved clinical outcomes. Prevention of recurrent episodes should be done by lifestyle modification and optimizing the lipid-lowering therapy. Periodic plasmapheresis can also be considered in non-complaint patients, but further studies are needed to support its clinical utility.

\section{Consent}

Witnessed informed consent taken from the patient to publish case report with images on May 23, 2016.

\section{Financial Support}

None.

\section{Conflicts of Interest}

None.

\section{References}

1. Cavallini G, Frulloni L, Bassi C, Gabbrielli A, Castoldi L, Costamagna G, De Rai P, et al. Prospective multicentre survey on acute pancreatitis in Italy (ProInf-AISP): results on 1005 patients. Dig Liver Dis. 2004;36(3):205211.

2. Toskes PP. Hyperlipidemic pancreatitis. Gastroenterol Clin North Am. 1990;19(4):783-791.

3. Fortson MR, Freedman SN, Webster PD, 3rd. Clinical assessment of hyperlipidemic pancreatitis. Am J Gastroenterol. 1995;90(12):2134-2139.

4. Tsuang W, Navaneethan U, Ruiz L, Palascak JB, Gelrud A. Hypertriglyceridemic pancreatitis: presentation and management. Am J Gastroenterol. 2009;104(4):984-991.

5. Scherer J, Singh VP, Pitchumoni CS, Yadav D. Issues in hypertriglyceridemic pancreatitis: an update. J Clin Gastroenterol. 2014;48(3):195-203.

6. Fredrickson DS. An international classification of hyperlipidemias and hyperlipoproteinemias. Ann Intern Med. 1971;75(3):471-472.

7. Goldberg AS, Hegele RA. Severe hypertriglyceridemia in pregnancy. J Clin Endocrinol Metab. 2012;97(8):25892596.

8. Berglund L, Brunzell JD, Goldberg AC, Goldberg IJ, Sacks F, Murad MH, Stalenhoef AF. Evaluation and treatment of hypertriglyceridemia: an Endocrine Soci- 
ety clinical practice guideline. J Clin Endocrinol Metab. 2012;97(9):2969-2989.

9. Kohli RS, Bleibel W, Shetty A, Dhanjal U. Plasmapheresis in the treatment of hypertriglyceridemic pancreatitis with ARDS. Dig Dis Sci. 2006;51(12):2287-2291.

10. Kadikoylu G, Yavasoglu I, Bolaman Z. Plasma exchange in severe hypertriglyceridemia a clinical study. Transfus Apher Sci. 2006;34(3):253-257.

11. Deng LH, Xue P, Xia Q, Yang XN, Wan MH. Effect of admission hypertriglyceridemia on the episodes of severe acute pancreatitis. World J Gastroenterol. 2008;14(28):4558-4561.

12. Tamez-Perez HE, Saenz-Gallegos R, Hernandez-Rodriguez K, Forsbach-Sanchez G, Gomez-de Ossio MD, Fernandez-Garza N, Zapata-de la Garza E, et al. [Insulin therapy in patients with severe hypertriglyceridemia]. Rev Med Inst Mex Seguro Soc. 2006;44(3):235-237.

13. Mikhail N, Trivedi K, Page C, Wali S, Cope D. Treatment of severe hypertriglyceridemia in nondiabetic patients with insulin. Am J Emerg Med. 2005;23(3):415-417.

14. Goldberg IJ. Lipoprotein lipase and lipolysis: central roles in lipoprotein metabolism and atherogenesis. $\mathrm{J} \mathrm{Li-}$ pid Res. 1996;37(4):693-707.

15. Weintraub M, Rassin T, Eisenberg S, Ringel Y, Grosskopf I, Iaina A, Charach G, et al. Continuous intravenous heparin administration in humans causes a decrease in serum lipolytic activity and accumulation of chylomicrons in circulation. J Lipid Res. 1994;35(2):229-238.

16. Jain P, Rai RR, Udawat H, Nijhawan S, Mathur A. Insulin and heparin in treatment of hypertriglyceridemia-induced pancreatitis. World J Gastroenterol. 2007;13(18):26422643.

17. Piolot A, Nadler F, Cavallero E, Coquard JL, Jacotot B. Prevention of recurrent acute pancreatitis in patients with severe hypertriglyceridemia: value of regular plasmapheresis. Pancreas. 1996;13(1):96-99. 